\title{
El recuperador celular y necesidad transfusional en pacientes intervenidos de cirugía cardíaca
}

\author{
Manuel Luque Oliveros
}

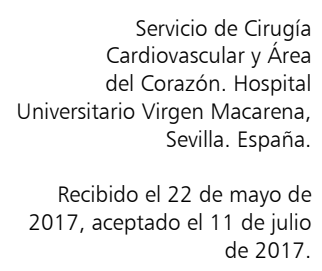

Correspondencia a: Manuel Luque Oliveros mluque.cec@gmail.com

\section{The cell saver and the transfusional need in patients undergoing cardiac surgery}

Objetive: Patients undergoing cardiac surgery are at high risk of being transfused with blood during the postoperative period, due to the decrease in haemoglobin and haematocrit levels. One of the alternatives to blood transfusion is the use of the intraoperative cell saver. The objective of this study was to identify whether the use of the intra-uremic cell saver decreases the transfusional rate during the immediate postoperative period. The immediate postoperative complications were also demonstrated in both groups. Material and Methods: Analytical, prospective study with two cohorts of patients distributed in control group (162) and intervention group (162). Sociodemographic variables, their diagnoses and surgical treatments, time of cardiac ischemia, haemoglobin, haematocrit, blood transfusion and haemorrhages, as well as variables specific to the cell saver were analyzed. The complications studied were; haemoglobinuria, fever, nausea and vomiting. The informed consent of all the patients was obtained and the data was submitted to the statistical package SPSS version 22.0. Results: The haemoglobin and haematocrit values of patients after surgery were different between groups (GC, $8.3 \mathrm{~g} / \mathrm{dl}, 22.8 \%$, GI, $10.4 \mathrm{~g} / \mathrm{dl}, 31.1 \mathrm{~g} \%$ ). Coinciding that the major transfused were those of the control group $(18.2 \%)$ versus the intervention group $(3.9 \%)$. The group that presented the greatest complication was the intervention group $(13.6 \%)$ who were those who used the cell saver. Hemoglobinuria $(82 \%)$ being the major complication. Conclusions: Patients who used the cell saver decreased the need for transfusion, but were the ones with the highest incidence of hemoglobinuria.

Key words: Cell saver; blood transfusion; cardiac.

\section{Resumen}

Objetivo: Los pacientes intervenidos de cirugía cardíaca presentan riesgo elevado de ser transfundidos con sangre durante el postoperatorio, debido al descenso de sus cifras de hemoglobina y hematocrito. Una de las alternativas a la transfusión sanguínea es el uso del recuperador celular intraquirúrgico. El objetivo de este estudio fue identificar si el uso del recuperador celular intraquirúgico disminuye la tasa transfusional durante el postoperatorio inmediato. También se ven las complicaciones postquirúrgicas inmediatas en ambos grupos. Material y Métodos: Estudio analítico, prospectivo con dos cohortes de pacientes distribuidos en grupo control (162) y grupo intervención (162). Se analizarón variables sociodemográficas, de sus diagnósticos y tratamientos quirúrgicos, tiempos de isquemia cardíaca, hemoglobina, hematocrito, transfusión sanguínea y hemorragias, así como variables propias del recuperador celular. Las complicaciones estudiadas fueron; hemoglobinuria, fiebre, náuseas y vómitos. Se obtuvo el consentimiento informado de todos los pacientes y se sometió los datos al paquete estadístico SPSS versión 22.0. Resultados: Las cifras de hemoglobina y hematocrito de los pacientes después de ser intervenido quirúrgicamente, fueron diferentes entre los grupos respectivamente (GC, 8,3 g/dL, 22,8\%. GI,10,4 g/dL, 31,1\%). Coincidiendo que los mayores transfundidos fueron los del grupo control $(18,2 \%)$ frente al grupo intervención $(3,9 \%)$. El grupo que presentó mayor complicación fue el grupo intervención $(13,6 \%)$ que eran los que utilizaron el recuperador celular. Siendo la hemoglobinuria (82\%) la mayor complicación. Conclusiones: Los pacientes que utilizaron el recuperador celular disminuyeron la necesidad transfusional, sin embargo, fueron los que mayor incidencia de hemoglobinuria padecieron.

Palabras clave: Recuperador celular; transfusión sanguínea; cardíaca. 


\section{Introducción}

Según the American Heart Association (AHA) ${ }^{1}$, la cirugía cardíaca, es una intervención quirúrgica compleja que lleva aparejado un riesgo elevado de transfusión sanguínea durante el postoperatorio. La transfusión, se define como el acto de administrar sangre completa o productos derivados de la misma (glóbulos rojos, plaquetas, plasma o crioprecipitados) procedente de un donante distinto al receptor ${ }^{2}$. Aún siendo consideradas salvadoras de vida a escala mundial, numerosos reportes y estudios de cohorte la han relacionado, con alteraciones inmunitarias e infecciones en el paciente durante el postoperatorio, con aumento de morbi-mortalidad a largo plazo ${ }^{3}$.

El reciente estudio de Vasudev y otros ${ }^{4}$, identificaron reacciones adversas transfusionales durante el postoperatorio, evidenciando; $83 \%$ hemoglobinuria, $50 \%$ fiebre, $66 \%$ hipotensión, $66 \%$ ictericia, $13 \%$ vómitos y con un sólo caso de hemólisis inmunológica. También, el estudio de Lelubre ${ }^{5}$, analizó a 2.872 pacientes transfundidos tras cirugía cardíaca, destacando que más de la mitad de ellos sufrieron, fallo cardíaco, fiebre y hemoglobina en orina durante las primeras horas del postoperatorio. A pesar de que existen datos que describen las reacciones adversas del acto de transfundir ${ }^{4,5}$, ésta debe ser considerada en cualquier ámbito de estudio, ya que su control inadecuado e ineficaz podría traer consecuencias graves para el paciente ${ }^{6}$.

En este sentido, the American College of Cardiology $(\mathrm{ACC})^{1}$, resalta la conveniencia de utilizar alternativas a la transfusión sanguínea, y con ello minimizar sus complicaciones y riesgos ${ }^{7}$, siendo en el contexto intraquirúrgico el uso del recuperador celular (RC), un dispositivo electrónico que se encarga de recuperar pérdidas sanguíneas del paciente en el campo quirúrgico, y que tras un ciclo de procesamiento, se obtiene un volumen de glóbulos rojos que se reinfunde de nuevo al paciente 8 .

La evidencia científica existente avala su efectividad y eficiencia ${ }^{6}$, sin embargo, the Blood Conservation Guideline ${ }^{9}$, señalaron que su uso no muestra perfil de seguridad, y por consiguiente podría influir en los trastornos de coagulación del paciente, entre otras, desencadenando ser transfundido. Ya, the Society of Thoracic Surgeons and the Society of Cardiovascular Anesthesiologists ${ }^{7}$, cuestionaron la eficacia del $\mathrm{RC}$, porque no abundan estudios comparativos y con amplias muestras, que determinen su incidencia. Además, recientes estudios publicados en 2016, afirmaron que el RC no es infalible, al no evitar en el $100 \%$ de los casos, la transfusión sanguínea, motivo por el cual, se pone en evidencia que hace falta más estudios, que ayuden a determinar sus indicaciones dentro del plano transfusiona ${ }^{10}$. Cabe resaltar, que la determinación de cuándo los beneficios del uso del RC sobrepasan el riesgo de las transfusiones sanguíneas, no está clara a partir de la evidencia disponible ${ }^{11}$, por ello nos preguntamos: ¿el uso del recuperador celular intraquirúrgico, disminuye la tasa de transfusión sanguínea de los pacientes durante el postoperatorio?

El presente estudio se justifica, por un lado, para aumentar la seguridad del paciente que va a ser intervenido quirúrgicamente, ya que la disminución de tasa transfusional, disminuyen sus riesgos asociados durante el postoperatorio. Además, de beneficiarse las instituciones hospitalarias, porque ahorrarían costes derivados de la transfusión. Por otro lado, para contribuir en el conocimiento científico de forma actualizada, la eficacia, efectividad y eficiencia del uso del recuperador celular en relación a la transfusión sanguínea, lo que sin duda merece la pena su estudio. En este contexto, se definió como objetivo; identificar si el uso del recuperador celular intraquirúgico sobre los pacientes intervenidos de cirugía cardíaca, disminuye la tasa transfusional durante el postoperatorio.

\section{Material y Métodos}

\section{Participantes}

Estudio analítico, observacional y prospectivo con dos cohortes de pacientes, en período comprendido de uno de marzo hasta el treinta y uno de octubre del año 2015 (ambos inclusive). Se incluyeron todos los pacientes programados e intervenidos de cirugía coronaria y valvular (aórtica, mitral y tricúspide) de nuestro hospital en dos quirófanos disponibles de cirugía cardíaca, en base a dos grupos: Grupo control (GC), donde se trató en un quirófano de forma convencional (drenaje aspirativo bajo presión), y grupo intervención (GI), donde, además, se utilizó el recuperador celular intraquirúrgico (Fresenius C.A.T.S ${ }^{\circledR}$ - Continuos Auto Transfusión System) en el otro quirófano.

Para ser incluidos todos los pacientes tenían que ser mayores de 18 años, participar de forma voluntaria en el estudio, tras explicación verbal y firma del consentimiento informado. Se excluyeron los pacientes que presentaban los siguientes criterios: intervenidos para implantación de un desfibrilador automático implantable (DAI), porque no eran considerados candidatos a recibir una transfusión sanguínea debido a la mínima pérdida de sangre durante la intervención. Reintervenidos o transfundidos 
$12 \mathrm{~h}$ antes de la cirugía, porque pueden alterar las cifras de hemoglobina y hematocrito enmascarando los datos reales recogidos. La preparación de los pacientes en ambos quirófanos, fue la misma y de manera convencional: anestesia general (inducción, mantenimiento y recuperación), canalización de vía periférica, radial, central de tres luces y catéter vesical transuretral de silicona con medidor de temperatura conectado al circuito extracorpóreo. El abordaje quirúrgico fue similar en todos los casos, tras esternotomía media longitudinal se procedió a la canulación de grandes vasos para la derivación cardiopulmonar mediante protocolo de heparina a tiempos de hemochron superior a 200 segundos, para evitar coagulación de sangre en la superficie sintética del propio circuito extracorpóreo. Se provocó cardiopléjico para realizar el procedimiento quirúrgico programado, siendo recuperadas las pérdidas sanguíneas por el aspirador seno del circuito extracorpóreo. Antes y después del uso del circuito extracorpóreo, se recuperó las pérdidas sanguíneas en el grupo control, con aspiradores conectados a un sistema de vacío bajo presión de $-20 \mathrm{~cm} \mathrm{H}_{2} \mathrm{O}$, cuyo volumen se desechaba. En el grupo intervención, se utilizó simultáneamente, aspiradores como el anterior grupo, y aspiradores propios del recuperador celular (RC).

El manejo del RC, fue similar en todos los pacientes, donde la sangre recuperada pasaba a un reservorio y tras un procesamiento de lavado (programa lavado de alta calidad, $2000 \mathrm{Rpm}$ ), se re infundía el volumen de glóbulos rojos obtenido, tras finalizar la cirugía. En ambos grupos, se comprobó la hemostasia de todas las anastomosis, y se revirtió la heparina, con su antagonista protamina ajustada a tiempos de hemochron. No hubo defunción en ambos grupos y al finalizar la cirugía todos los pacientes presentaban niveles hematológicos dentro del rango de normalidad. Se recolectaron las siguientes variables en el período preoperatorio: Edad, sexo, índice de masa corporal (IMC), diagnóstico médico, procedimiento quirúrgico, hemoglobina (g/Dl) y hematocrito (\%), doce horas antes de acudir el paciente a quirófano, a través de la historia clínica digitalizada del paciente. En el período intraoperatorio se recogieron variable de tiempo de isquémia cardíaca (minutos) y variables del RC: Volumen de sangre rescatada/procesada y volumen de glóbulos rojos reinfundido (todas expresado en $\mathrm{Ml}$ ), al finalizar la cirugía programada, cuyos datos fueron obtenidos desde la pantalla digital del propio RC. En el período postoperatorio las variables recogidas fueron: Hemoglobina (g/Dl), hematocrito (\%), hemorragias y complicaciones tales como hemoglobinuria (ex- tracción de muestra de orina del urinómetro), fiebre y náuseas/vómitos $(\%)$, durante las doce primeras horas de sus estancia en UCI. Todos los datos se recogieron a través de la historia clínica digitalizada.

De los 478 pacientes programados para someterse a cirugía cardíaca, finalmente se seleccionaron 324 pacientes por cumplir con los criterios de inclusión y firmado el informe de consentimiento informado, de los cuales 162 correspondían a grupo control y 162 a grupo intervención. Los datos de estos pacientes se obtuvieron a través de la solicitud de gestión de la demanda. El proyecto se desarrolló en todo momento de acuerdo con los principios de la Declaración de Helsinki de 1975 con la revisión de octubre de 2000 y Ley Orgánica 15/1999 de 13 de diciembre). Todos los participantes fueron informados verbalmente de las características del estudio, así como la privacidad de sus datos personales en el tratamiento de la información. Todos los registros y consentimiento informado por escrito de cada participante, se almacenó bajo un número asignado, donde sólo el investigador tenía acceso a ello. Este proyecto fue revisado y aprobado por la Comisión de Ética e Investigación Clínica de nuestro Hospital.

\section{Análisis de datos}

Para las variables cuantitativas se expresaron como variable continua, utilizando media (desviación estándar) según sea apropiado. Además, se utilizó prueba de Chi-cuadrado de Pearson para las variables categóricas. Para comparar las variables relacionadas e independientes se usó la prueba de $\mathrm{T}$ de Student. Todos los valores de $\mathrm{p}$ tuvieron un nivel de significación estadística de $\mathrm{p}<0,05$. El análisis estadístico se realizó bajo el paquete estadístico SPSS, versión 22.0.

\section{Resultados}

De los 478 pacientes programados para cirugía cardíaca, $324(67,7 \%)$ fueron incluidos en este estudio, de los cuales $162(33,85 \%)$ pertenecen al GC y $162(33,85 \%)$ al GI. Se excluyeron un total de 154 $(32,3 \%)$ pacientes por adecuarse a criterios de exclusión. $146(30,5 \%)$ se operaron de DAI y $8(1,6 \%)$ no firmaron el consentimiento informado. El perfil de los participantes en ambos grupos se visualizan conforme la siguiente Tabla 1.

La media de los valores de hemoglobina y hematocrito en ambos grupos fueron similares en el período preoperatorio. Sin embargo, existió diferencias estadísticamente significativa durante el período postoperatorio, donde el grupo control sufrió 
un descenso de sus cifras respecto al otro grupo, lo que justifica su aumento de transfusión sanguínea y hemorragias, conforme indica la Tabla 2.

La media de valores del tiempo de isquemia cardíaca fue similar en ambos grupos (GC;82', GI: 79') $\mathrm{p}=0,27$. Y se utilizó el RC bajo el epígrafe de lavado de alta calidad con $2000 \mathrm{Rpm}$ en todos los casos del GI $(\mathrm{N}=162)$. Se rescató y procesó un volumen medio de $1.500 \mathrm{ml}$ de sangre autóloga (máximo de $1.900 \mathrm{ml}$ y un mínimo de $151 \mathrm{ml}$ ). Del procesamiento, se obtuvieron y reinfundieron un volumen medio de $600 \mathrm{ml}$ de glóbulos rojos (máximo de $710 \mathrm{ml} \mathrm{y}$ un mínimo de $100 \mathrm{ml}$ ).

Por último, el grupo que presentó mayores complicaciones durante el postoperatorio, fue el grupo intervención, destacando la hemoglobinuria como la principal complicación, conforme la Tabla 3.

\section{Discusión}

The American Heart Association (AHA) ${ }^{12}$, declaran que $\mathrm{Hb} 12$ g/Dl y Htco 28\% son cifras aceptables en la práctica de la cirugía cardíaca. Una posición validada por otros estudios ${ }^{13}$. En nuestro caso la $\mathrm{Hb}$ y Htco fueron inferiores en ambos grupos durante el postoperatorio inmediato, coincidiendo con el reciente ensayo the Transfusión Indication Thershold Reduction (Titre2), donde $\mathrm{Hb}$ inferior a $9 \mathrm{~g} / \mathrm{Dl}$ y Htco inferior a $27 \%$, son firmes candidatos a ser transfundidos con sangre o hemoderivados ${ }^{14}$. Existen evidencias científicas que detallan que es debido a procedimientos secundarios del circuito extracorpóreo, y a prolongados tiempos de isquemia cardíaca $^{15}$, pero nuestros grupos han tenido mismos tratamientos quirúrgicos y tiempos de isquemia, obteniendo diferencias significativas entre los grupos durante el postoperatorio, lo que sin duda despierta interés su significado. Por un lado, conocer el umbral transfusional se vuelve un tema controvertido, porque cada protocolo está en consonancia con criterios propios de la institución sanitaria y del profesional encargado según evidencia existente. Y por otro, el manejo del RC, que a pesar de la evidencia científica posicionarlo como alternativa a la transfusión sanguínea, sigue siendo actualmente un tema de controversia entre los expertos ${ }^{16}$. Un estudio de Steiner ${ }^{17}$, sostiene que en aquellos pacientes donde no se utilicen estrategias alternativas a la transfusión sanguínea, aumenta el riesgo de recibirlas. Mismas conclusiones aparecen en otros estudios ${ }^{18,19}$. Sin embargo, a pesar de que coincide con nuestros resultados, no comparan con grupos de pacientes que si la utilizan, como se detalla en nuestra investigación.

En relación a lo anterior, destacar otros estudios
Tabla 1. Características relacionales de los participantes

\begin{tabular}{|lccc|}
\hline Variables & $\begin{array}{c}\text { GC (n= 162) } \\
\text { M (DE) }\end{array}$ & $\begin{array}{c}\text { GI (n= 162) } \\
\text { M (DE) }\end{array}$ & p* $^{*}$ \\
Perfil & $66(9,3)$ & $65(9,7)$ & 0,56 \\
Edad (años) & & & \\
Sexo (\%) & $130(80,6)$ & $144(89,4)$ & 0,09 \\
$\quad$ Hombres & $32(19,4)$ & $17(10,6)$ & \\
$\quad$ Mujeres & $23(5,3)$ & $20 \quad(5,7)$ & 0,26 \\
IMC $^{\text {b }}$ & & & 0,31 \\
Diag./IQ & 66 & 68 & \\
Aórtica (\%) & 18 & 16 & \\
Arterioesclerosis coronaria (\%) & 14 & 3 & \\
Mitral (\%) & 2 & 13 & \\
Tricúspide (\%) & & 3 & \\
\hline
\end{tabular}

${ }^{\mathrm{a}} \mathrm{M}(\mathrm{DE})=$ media (desviación estándar); ${ }^{\mathrm{b}} \mathrm{IMC}=$ índice de masa corporal. ${ }^{\mathrm{c} D i a g} . /$ $\mathrm{IQ}=$ diagnósticos médicos/intervenciones quirúrgicas programadas. Entre los grupos, no existieron diferencias estadísticamente significativas $*(p<0,05)$.

Tabla 2. Valores de hemoglobina, hematocrito, hemorragias y transfusión de los participantes

\begin{tabular}{|lccc|}
\hline Variables & $\begin{array}{c}\text { GC (N= 162) } \\
(\mathbf{M}) \mathbf{a}\end{array}$ & $\begin{array}{c}\text { GI (N= 162) } \\
(\mathbf{M})\end{array}$ & $\mathbf{p}$ \\
Preoperatorio & & & \\
Hemoglobina (g/D1) & 12,1 & 12,3 & 0,38 \\
Hematocrito (\%) & 36,9 & 39,2 & 0,36 \\
Postoperatorio & & & \\
Hemoglobina (g/D1) & 8,3 & 10,4 & $0,02^{*}$ \\
Hematocrito (\%) & 22,8 & 31,1 & $0,04^{*}$ \\
Hemorragias (\%) & 13,6 & 8,1 & $0,04^{*}$ \\
Transfusión sanguínea (\%) & 18 & 3,9 & $0,007^{*}$ \\
\hline
\end{tabular}

${ }^{\mathrm{a}} \mathrm{M}=$ media; Existió diferencias estadísticamente significativas entre los grupos durante el período postoperatorio ${ }^{*}(\mathrm{p}<0,05)$.

Tabla 3. Complicaciones de los participantes durante el postoperatorio

\begin{tabular}{|lccc|}
\hline Variables & $\begin{array}{c}\text { GC }(\mathbf{N}=\mathbf{1 6 2}) \\
(\mathbf{M})^{\mathbf{a}}\end{array}$ & $\begin{array}{c}\text { GI }(\mathbf{N}=\mathbf{1 6 2}) \\
(\mathbf{M})\end{array}$ & $\mathbf{p}$ \\
Complicaciones & 8,1 & 13,6 & $0,04^{*}$ \\
Hemoglobinuria (\%) & 68 & 82 & \\
Fiebre (\%) & 25 & 15 & \\
Náuseas y vómitos (\%) & 7 & 3 & \\
\hline
\end{tabular}

${ }^{\mathrm{a}} \mathrm{M}=$ media; Existió diferencias estadísticamente significativas entre los grupos durante el período postoperatorio ${ }^{*}(\mathrm{p}<0,05)$, siendo la mayor complicación la hemoglobinuria en el grupo intervención.

recientes, que trataron de correlacionar los cambios en la oxigenación cerebral durante la terapia de transfusión sanguínea y reinfusión de glóbulos rojos procedente de RC, no pudiendo observar diferencias entre ambos ${ }^{20}$. Aunque otros estudios de cohorte, demuestran que para disminuir la transfusión san- 
guínea con el uso del RC, éste debe mantener su uso durante el postoperatorio ${ }^{21}$, lo que se aleja de nuestro propósito de alternativas intraquirúrgicas para la disminución de transfusión sanguínea durante el postoperatorio inmediato.

En el contexto de otras cirugías, el estudio Orthopedic Surgery Transfusion Hemoglobin European Overview (OSTHEO) ${ }^{22}$, demuestran que el riesgo de recibir transfusión sanguínea está presente en aquellos pacientes que no han utilizado estrategias alternativas, pero no justifica ni el por qué, ni tan siquiera la comparan con aquellos que sí la utilizaron. Del mismo modo, otros estudios ${ }^{23}$, analizan las estadísticas de las complicaciones del acto de transfundir, pero no la proporcionan derivada al uso del RC, e incluso, el estudio de Rebarber ${ }^{24}$, que no pudo demostrar cuáles y por qué aumentaron las complicaciones del paciente después de recibir la reinfusión de glóbulos rojos procedente del RC.

No obstante, existen datos que declaran que la reinfusión de glóbulos rojos procedente del $\mathrm{RC}$ sin plaquetas, plasma, ni factores de coagulación, mitiga efectos negativos de la función hematológica del paciente, lo que justifica que en estos pacientes tengan una necesidad transfusional, sobre todo al consumo de factores de coagulación ${ }^{25}$, ya que provoca una sensibilización plaquetaria significativa, pero insistimos que no establecen un marco comparativo con aquellos pacientes que recibieron la transfusión sanguínea, por tanto coincidimos con otros estudios que postulan que toda investigación que se encauce en el uso de los recuperadores celulares como medida a la transfusión sanguínea, merecen precaución en cuanto su utilización de forma generalizada, por lo que requieren estudios más amplios para confirmar sus resultados ${ }^{26}$.

Esta falta de evidencia científica actualizada, está originando entre los profesionales que manejan estos dispositivos, incertidumbre y cuestionan encuadrarlo como alternativa a la transfusión sanguínea. Como recomendación basada en nuestros resultados, el uso del RC intraquirúrgico, hace que el paciente disminuya la necesidad transfusional durante el postquirúrgico, en comparación con aquellos pacientes que no la utilizan. La explicación de estos resultados es que la reinfusión de glóbulos rojos procedente del RC, aumenta las cifras de $\mathrm{Hb}$ y Htco lo que, a su vez, disminuye el riesgo de transfusión. Sin embargo, esta experiencia recomienda encarecidamente que en aquellas cirugías que se prevee pérdidas sanguíneas considerables intraquirúrgicas, y que utilicen medidas alternativas a la transfusión sanguínea como el RC, también tendrá complicaciones derivadas de su uso, como la hemoglobinuria, por lo que te permite tratarla con antelación, además de otros posibles eventos adversos derivados del mismo durante el período postquirúrgico inmediato.

Consideramos que este estudio contribuye a la seguridad del paciente porque ofrece al profesional sanitario información de las consecuencias derivados del uso del RC, lo que sin duda se verán beneficiados las instituciones hospitalarias, porque ahorrarían costes derivados del mismo. Por último, insistimos en la necesidad de realizar más estudios comparativos con grandes muestras de participantes destinados a evaluar las complicaciones exhaustivas del paciente durante el postoperatorio, derivados del uso y no del RC, y no solo limitarlo a la disminución de las transfusiones sanguíneas. Lo cual, hace posicionarnos con la $\mathrm{AHA}$ y $\mathrm{ACC}^{1}$ cuando inciden que cualquier estudio que muestren resultados satisfactorios debe ser investigado a fondo, por aumentar no solo la calidad de los servicios prestados, sino también para dar continuidad a los cuidados ofrecidos. Por ello, se concluye que los pacientes que utilizaron el recuperador celular disminuyeron la necesidad transfusional, sin embargo, fueron los que mayor incidencia de hemoglobinuria padecieron.

\section{Responsabilidades éticas}

Protección de personas y animales. Los autores declaran que para esta investigación no se han realizado experimentos en seres humanos ni en animales.

Confidencialidad de los datos. Los autores declaran que han seguido los protocolos de su centro de trabajo sobre la publicación de datos de pacientes.

Derecho a la privacidad y consentimiento informado. Los autores han obtenido el consentimiento informado de los pacientes y/o sujetos referidos en el artículo. Este documento obra en poder del autor de correspondencia.

\section{Financiación}

El presente trabajo no posee fuente de financiación.

\section{Conflicto de intereses}

El autor declara no tener ningún conflicto de intereses.

\section{Agradecimientos}

Al estadístico Dr. Polo por su gran ayuda prestada. 


\section{Bibliografía}

1. Nishimura RA, Otto CM, Bonow RO, Carabello BA, Erwin JP, Guyton RA, et al. 2014 AHA/ACC guideline for the management of patients with valvular heart disease: a report of the American College of Cardiology/ American Heart Association Task Force on Practice Guidelines. J Am Coll Cardiol. 2014;63:57-185. Doi: 10.1016/j. jacc.2014.02.536.

2. Flórez S, Prieto L, Maroto L, Bustamante J, Cubero T, Fernández M, et al. Blood and surgery. Saving strategy in cardiac surgery. Span J Surg Res. 2011;14:89-114.

3. Weightman WM, Gibbs NM. Management of coagulation: an Australian perspective. C Opin Anaesth. 2012;25:86-95. Doi: 10.1097/ACO.0b013e32834e46b1.

4. Vasudev R, Sawhney V, Dogra M, Raj TR. Transfusion-related adverse reactions: From institutional hemovigilance effort to National Hemovigilance program. Asian J Transfus Sci. 2016;10:31-36. Doi: 10.4103/0973-6247.175391.

5. Lelubre C, Vicent JL. Relationship betwewn red cell storage duration and outcomes in adults receiveng red cell transfusions: a systematic review. Crit Care. 2013;17:66. Doi: 10.1186/cc12600.

6. Pérez-Valdivieso JR, Monedero P, GarcíaFernández N, Vives M, Lavilla FJ, BesRastrollo M, et al. Blood transfusión during heart surgery. A retrospective nested case-control. Rev Esp Anesthe Rean. 2013;60:79-86. Doi:10.1016/j. redar.2012.10.002.

7. Ferraris VA, Brown JR, Despotis GJ, Hammon JW, Reece TB, Saha SP et al. 2011 update to the Society of Thoracic Surgeons and the Society of Cardiovascular Anesthesiologists blood conservation clinical practice guidelines. Ann Thorac Surg. 2011;9:944-982. Doi: 10.1016/j.athoracsur.2010.11.078.

8. Pérez A, Gredilla E, De Vicente J, Sánchez F, Gilsanz F. Implementation of a patient blood management program in pediatric scolisis surgery. Rev Esp Anestesiol Reanim. 2016;63:69-77. Doi: 10.1016/j.redar.2015.04.009.

9. Jonas RA. Blood conservation guidelines for pediatric patients. Ann Thorac
Surg. 2011;91:403-404. Doi:10.1016/j. athoracsur.2011.03.056.

10. Ortiz G, Ariza F, Trujillo A, Bejarano A, Gutiérrez JM, Gálves K, et al. Manejo del sangrado y la coagulación en la práctica clínica. Evaluación de la evidencia y recomendaciones mediante estrategia GRADE. Primera reunión de expertos. Acta Colomb Cuid Intensivo. 2016;16: 172- 194. Doi:10.1016/j.acci.2016.04.001.

11. Gakhar H, Bagouri M, Bommireddy R, Klezl Z. Role of Intraoperative Red Cell Salvage and Autologus Transfusion in Metastatic Spine Surgery: A pilot Study and Review of Literature. Asian Spine J. 2013;7:167-172. Doi: 10.4184/ asj.2013.7.3.167.

12. Mozaffarian D, Benjamin EJ, Go AS, Arnett DK, Blaha MJ, Cushman M, et al. Heart Disease and Strole Statistics-2016 Update: A Report From the American Heart Association. Circulation. 2016;133:38-360.Doi: 10.1161/ CIR.0000000000000350.

13. Drouet N. European transfusion practices: the SANGUIS survey. Cah Anesthesiol. 1994;42:425-8.

14. Murphy J, Pike K, Rogers CA, Wordsworth S, Stokes EA, Angelini GD et al. Liberal or restrictive transfusion after cardiac surgery. N Engl J Med. 2015;372:997-1008. Doi: 10.1056/ NEJMoa1403612.

15. Pérez A, Gredilla E, De Vicente J, Navarro R, Gilsanz F. Characteristics and quality of intra-operative cell salvage in paediatric scoliosis surgery. Rev Esp Anestesiol Reanim. 2016;63:78-83. Doi: 10.1016/j.redare.2015.10.003.

16. Naranjo A, Husein E, Marcano LE, Rivera K, Aguilera E, Carballés F. Veintitrés años de tratamiento quirúrgico al adulto con cardiopatía congénita. Rev Cubana Cardiol. 2013;19:17-20.

17. Steiner ME, Ness PM, Assmann SF, Triulzi DJ, Sloan SR, Delaney M et al. Effects of red-cell storage duration on patients undergoing cardiac surgery. N Engl J Med. 2015;372:1419-1429. Doi: 10.1056/NEJMoa1414219.

18. Martini J, Maisch S, Pilshofer L, Streif W, Martini W, Fries D. Fibrinogen concentrate in dilutional coagulopathy: a dose study in pigs. Transfusion.
2014;54(1):149-157. Doi: 10.1111/ trf. 12241

19. Kozek-Langenecker SA, Afshari A, Albaladejo P, Santullano CA, De Robertis E, Filipescu D et al. Management of severe perioperative bleeding: guidelines from the European Society of Anaesthesiology. Eur J Anaesthesiol. 2013;30:270-382. Doi: 10.1097/ EJA.0b013e32835f4d5b.

20. Hakim M, Tumin D, Martin DP, Samora W, Beebe AC, Klamar JE, et al. Chances in Cerebral Oxygenation during Transfusion Therapy. J Extra Corpor Technol. 2016;48:173-178.

21. Vonk AB, Meesters MI, Garnier RP, Romijn JW, van Barneveld LJ, Heymans MW, et al. Intraoperative cell savage is associated with reduce postoperative blood loss and transfusion requirements in cardiac surgery: a cohort study. Transfusion. 2013;53:2782-2789. Doi:10.1111/ trf. 12126 .

22. Rosencher N, Kerkkamp HE, Macheras G, Munuera LM, Menichella G, Barton DM, et al. Orthopedic Surgery Transfusion Hemoglobin European Overview (OSTHEO) study: blood management in elective knee and hip arthroplasty in Europe. Transfusion. 2003;43:459-469.

23. Lacroix L, Hébert PC, Fergusson DA, Tinmouth A, Cook DJ, Marshall JC, et al. Age of transfused blood in critically ill adults. N Engl J Med. 2015;372:1410-8. Doi: 10.1056/NEJMoa1500704.

24. Rebarber A, Lonser R, Jackson S, Copel JA, Sipes S. The safety of intraoperative autologous blood collection and autotransfusion during caesarean section. Am J Obstet Gynecol.1998;179:715-20.

25. Gäbel J, Malm CJ, Radulovic V, Shams Hakimi C, Westerberg M, Jeppsson A. Cell saver processing mitigates the negative effects of wound blood on plaquetelet function. Acta Anaesthesiol Scand. 2016;60:901-909. Doi. 10.1111/ aas. 12730 .

26. Al-Riyami AZ, Al-Khabori M, Baskaran B, Siddiqi M, Al-Sabti H. Intra-operative cell salvage in cardiac surgery may increase platelet transfusión requirements: a cohort study. Vox Sang. 2015;109:280286. Doi: 10.1111/vox.12280. 